\title{
Sediment dynamics. Part 2. Dune formation in pipe flow
}

\author{
MALIKA OURIEMI, PASCALE A USSILLOUS, \\ AND ÉLISABETH GUAZZELLI \\ IUSTI CNRS UMR 6595 - Polytech'Marseille - Aix-Marseille Université (U1), \\ 5 rue Enrico Fermi, 13453 Marseille cedex 13, France
}

(Received 22 April 2009)

We present a phase diagram of the different dune patterns observed when a bed composed of spherical particles is subjected to a pipe flow. While the threshold for incipient motion is determined by the Shields number, that for dune formation seems to be controlled by the Reynolds number. A simple linear stability analysis based on a particle flux derived by Ouriemi, Aussillous \& Guazzelli (2009) accounts reasonably well for the experimental observations.

\section{Introduction}

A very common feature that arises when bed constituted of particles are submitted to shearing flows is the formation of ripples, i.e. small waves on the bed surface having wavelengths scaling with the particle size, or of dunes, i.e. larger mounds or ridges having wavelengths comparable to or larger than the fluid height.

Since the seminal work of Kennedy (1963), the prevalent mechanism for dune or ripple formation is the fluid inertia or more precisely the phase-lag between the bottom shear stress and the bed waviness generated by the fluid inertia. In that case, the shear stress, the maxima of which are slightly shifted upstream of the crests, drags the particles from the troughs up to the crests. This destabilising mechanism seems to be robust enough to apply to any steady flow, either turbulent, see e.g. Engelund (1970), Richards (1980), Sumer \& Bakioglu (1984), Colombini (2004) and Claudin \& Andreotti (2006), or viscous, see e.g. Charru \& Mouilleron-Arnould (2002), and Charru \& Hinch (2006).

A few stabilising mechanisms have been proposed, among which a well identified stabilising effect due to the inclination of the bed, see e.g. Fredsøe (1974), Richards (1980), Sumer \& Bakioglu (1984), Charru \& Mouilleron-Arnould (2002), Charru \& Hinch (2006) and Charru (2006). For nonzero slope, the gravity force parallel to the bed surface favours the downhill motion of the particles and conversely impedes the uphill motion. Another stabilising mechanism related to particle inertia was also suggested to arise from the phase-lag between the bottom shear stress and the particles flow rate. This effect can be expressed in term of an inertial length, see e.g. Andreotti, Claudin \& Douady (2002), or a deposition length coming from a stabilising crest-erosion process, see e.g. Charru \& Hinch (2006) and Charru (2006).

However, a complete description of the bed instability is still lacking as the coupling between the granular media and the fluid is poorly understood. Usually, one first calculates the fluid flow as if the wavy bottom were fixed by considering the superposition of a base flow on the flat bed and a perturbation induced by the wavy bottom. Then, one needs to relate the particle flow rate to the calculated shear stress at the top of the bed. 


$$
\begin{array}{ccc}
\text { Authors } & \text { Flow } & \text { Threshold Prediction } \\
\text { Sumer \& Bakioglu (1984) } & \text { turbulent } & R e_{p}=\frac{d u_{*}}{\nu}=10-26 \\
\text { Charru \& Mouilleron-Arnould (2002) } & \text { laminar } & \theta=\theta_{0}^{c}\left(\frac{30}{\theta_{0}^{c} G a \mu}\right)^{1 / 2} \frac{d}{h_{f}} \\
\text { Charru \& Hinch (2006) } & \text { laminar } & G a=\frac{396}{\theta_{0}^{c}}\left(\frac{d}{h_{f}}\right)^{3}
\end{array}
$$

TABLE 1. Stability analysis prediction for the instability threshold. The particle Reynolds number is $R e_{p}$ (with $d$ the particle size, $u_{*}$ the shear velocity, and $\nu$ the kinematic viscosity of the fluid). The Shields number, $\theta$, is constructed as the ratio of the shear stress at the top of the bed to the apparent weight of a single particle. The critical Shields number for incipient particle motion is $\theta_{0}^{c}$, the friction coefficient $\mu$, and the fluid height is $h_{f}$. The Galileo number $G a=d^{3}\left(\rho_{p}-\rho_{f}\right) g / \nu$ (with $\rho_{p}$ and $\rho_{f}$ the particle and fluid density, respectively) is the Reynolds number based on the Stokes settling velocity of the particles.

Particle mass conservation equation is finally solved to provide the linear growth rate of the instability. Several algebraic law relating the particle flow rate to the bottom shear stress have been proposed in the literature, see tables 1 and 2 of Ouriemi, Aussillous \& Guazzelli (2009), leading to different control parameters for the instability threshold, such as the Reynolds, Shields, or Galileo numbers, see table 1 where some predictions of linear stability analyses, which are of particular interest for the present study, are presented.

There is not yet a complete experimental proof that this type of modelling captures the essence of the instability. The stability analyses of Sumer \& Bakioglu (1984) and Charru \& Mouilleron-Arnould (2002) having the particle transport described by an algebraic law, a Bagnold-type law and a cubic law respectively, have found preferred initial wavelengths to be an order of magnitude smaller than the observed dune lengths. A recent stability analysis by Charru (2006) seems to improve the predictions by including a phase-lag which erodes the peaks, i.e. the additional stabilisation mentioned above.

The present contribution aims at investigating dune formation in a confined, wellcontrolled flow, namely a flow in a closed pipe. In $\S 2$, we present an experimental study of the evolution of a particle bed in a pipe flow. Different dune patterns are observed as the flow rate is increased from the laminar to the turbulent regimes. We then focus on the threshold for destabilisation of the flat bed leading to dune formation. In $\S 3$, we adapt the stability analysis of Charru \& Hinch (2000) to a Poiseuille flow and choose to use simply the algebraic law relating the particle flux to the cube of the Shields number found by Ouriemi, Aussillous \& Guazzelli (2009). The control parameter of the instability is the Reynolds number and the predicted wavelength at onset scales with the fluid thickness. In $\S 4$, a phase diagram for the different dune patterns observed is proposed and comparison with theoretical predictions provided. 


$\begin{array}{ccccc}\text { Batch } & \text { Composition } & d(\mu \mathrm{m}) & \rho_{p}\left(\mathrm{~g} / \mathrm{cm}^{3}\right) & \text { Supplier } \\ \text { A } & \text { Glass } & 132 \pm 22 & 2.490 \pm 0.003 & \text { Potters-Ballotini } \\ \text { B } & \text { Polystyrene } & 538 \pm 24 & 1.051 \pm 0.002 & \text { Maxi-Blast } \\ \text { C } & \text { PMMA } & 193 \pm 30 & 1.177 \pm 0.002 & \text { Lehmann \& Voss \& Co. }\end{array}$

TABle 2. Particle characteristics. The particle density, $\rho_{p}$, was measured with a pycnometer and a fluid of measured density. The particle size distributions were determined with a digital imaging system. The mean diameter is noted $d$ and the error corresponds to one standard deviation.

\begin{tabular}{ccc} 
Fluid & $\eta(\mathrm{cP})$ & $\rho_{f}\left(\mathrm{~g} / \mathrm{cm}^{3}\right)$ \\
\hline 1 & $0.7 \pm 1$ & $0.999 \pm 0.001$ \\
2 & $6.7 \pm 1$ & $1.016 \pm 0.001$ \\
3 & $8 \pm 1$ & $1.018 \pm 0.001$ \\
4 & $10 \pm 1$ & $1.022 \pm 0.001$ \\
5 & $12 \pm 1$ & $1.023 \pm 0.001$
\end{tabular}

TABLE 3. Fluid characteristics at $T=35^{\circ} \mathrm{C}$. The viscosity, $\eta$, was measured with a falling ball viscometer and the fluid density, $\rho_{f}$, with a pycnometer.

\section{Experimental observations}

\subsection{Experimental set-up}

The experimental test section was a horizontal glass tube of inner diameter $D=3 \mathrm{~cm}$ and length $L=1.8 \mathrm{~m}$. The measurement zone was located at $\approx 50 \mathrm{~cm}$ from the entrance. This length corresponded to the entry length necessary for the laminar flow to develop fully inside the tube at $R e_{\text {pipe }} \approx 250$, where $R e_{\text {pipe }}=4 Q_{\text {pipe }} \rho_{f} / \pi D \eta$ is the tube Reynolds number with $Q_{\text {pipe }}$ the flow rate, $\rho_{f}$ the fluid density, and $\eta$ the viscosity. Experiments were performed in the range $10^{-1} \lesssim R e_{\text {pipe }} \lesssim 10^{4}$. For $R e_{\text {pipe }} \lesssim 250$, the laminar flow was then fully developed in all the measurement zone while it was not for $250 \lesssim R e_{\text {pipe }} \lesssim$ 2500. The transition toward turbulence occurred for $R e_{\text {pipe }} \approx 2500$.

Three different batches of spheres and five different mixtures of distilled water and UCON oil 75H-90000 were used in the experiments as indicated in tables 2 and 3. First, the tube was filled with fluid and the particles were carefully introduced to form an uniform flat bed. Secondly, in order to avoid flow perturbations from a pump, the flow was driven by gravity. The fluid was delivered to the tube by continuous overflow from an overhead tank of variable height. At the outlet of the tube, the particles were captured by a mesh while the fluid was run into a reservoir. A pump carried the fluid back to the overflowing tank. The temperature of the fluid was maintained at $35 \pm 1^{\circ} \mathrm{C}$ by using a thermostated bath as a fluid reservoir in the fluid circulating loop. To measure $Q_{\text {pipe }}$, we collected a given volume of fluid at the outlet of the tube in a given time. It is worth mentioning that, even though the flow is controlled by gravity, the pressure losses in the flow loop result in an imposed flow-rate in the test section despite the changes in the tube section as particles are lost.

\subsection{Classification of dune patterns}

Five different regimes can be observed as summarised in figure 1 for two different batches of particles (batches A and C) and using fluids of varying viscosity to explore the full 
$R e_{\text {pipe }}$ range. For convenience, we have chosen to represent their domain of validity as a function of $R e_{\text {pipe. }}$. Below the critical Shields number for incipient particle motion, $\theta_{0}^{c} \approx 0.12$ (the 0 subscript indicates that this threshold corresponds to a flat bed), the bed shape does not evolve, see Charru, Mouilleron-Arnould \& Eiff (2004), Loiseleux, Gondret, Rabaut \& Doppler (2005), and Ouriemi, Aussillous, Medale, Peysson \& Guazzelli (2007). Note that this first threshold is determined by the Shields number and not the Reynolds number. Above this first threshold, we observe a second regime where the bed shape evolves but without dune formation. In this regime, a few layer of particles are set in motion at the surface of the bed by the fluid flow. The bed shape evolves, becomes slightly tilted and the bed height decreases with increasing time as the test section is not fed in with particles. When the experiment is run for a long enough time, the bed shape eventually freezes, exhibiting a flat surface.

Increasing again $R e_{\text {pipe }}$, three regimes exhibiting different dune patterns can be found. For the first dune regime, which only exists in laminar flow, the dunes denoted 'small dunes' present small amplitudes. The second dune regime is observed either in laminar or turbulent flow. In this regime, the dunes are characterised by the existence of vortices located at their front as can be seen in online movie 1 . The vortices erode the particle bed (see figure 1 top view of the vortex dunes). This thus leads to dunes of very large amplitude denoted 'vortex dunes'. Finally, when the flow is turbulent (and only in that case), we observe a new dune pattern that we called 'sinuous dune'. The bottom photograph of figure 1 shows a top view of a 'sinuous dune'. The particle bed is eroded asymmetrically leading to the formation of a pattern having a double periodicity. This may be explained by a destabilisation of the initial vortices observed in the 'vortex dunes'.

\subsection{Evolution of dune amplitude, wavelength, and phase velocity}

To quantify more precisely the time-evolution of the dunes, we recorded the evolution of the bed height as a function of time, for a combination of fluid and particles, a given flow rate $Q_{\text {pipe }}$, and an initial height of the bed, $h_{p}^{\text {start }}$. Using the same experimental technique as in Ouriemi, Aussillous \& Guazzelli (2009) where further details can be found, the bed height was measured by imaging the upper layer of the bed illuminated by a laser sheet aligned with the tube length in its middle. The measurement zone spanned over 45 or $75 \mathrm{~cm}$ and started at $\approx 50 \mathrm{~cm}$ from the entrance of the tube. The use of such a large measurement zone provided a precise study of the wavelength evolution over a long time but leaded to a poor resolution in dune amplitude as the accuracy in height was $\approx 2-3 \mathrm{~mm}$.

Here, we focus on the two first dune regimes, i.e. the regimes of small and vortex dunes. The spatio-temporal evolution of the bed height for three experiments are plotted in figure 2 , in the small dune regime (a) and in the vortex dune regime (b) and (c), with bed heights coded in grey scale. In both regimes, we observe the formation of initial dunes in the measurement zone. These dunes have a relatively uniform wavelength and move in the flow direction inside the tube. However, the initial dunes preceding the vortex dunes differ from the small dunes as will be evidenced later.

In the case of vortex dunes, the flow perturbation at the entrance of the tube generates a first vortex dune whose vortices create a second dune downstream and so on. The vortex perturbation by propagating downstream creates dunes downstream over the entire length of the tube which progressively take over the initial dunes, see the top plots of figure 2 (b) and (c). At the same time, the dune pattern moves downstream as the dunes themselves are moving in the flow direction. With increasing time, the vortices between two adjacent dunes erode the particle bed and the dunes are separated by regions emptied of particles. Even though no dune coalescence is observed, the pattern wavelength 


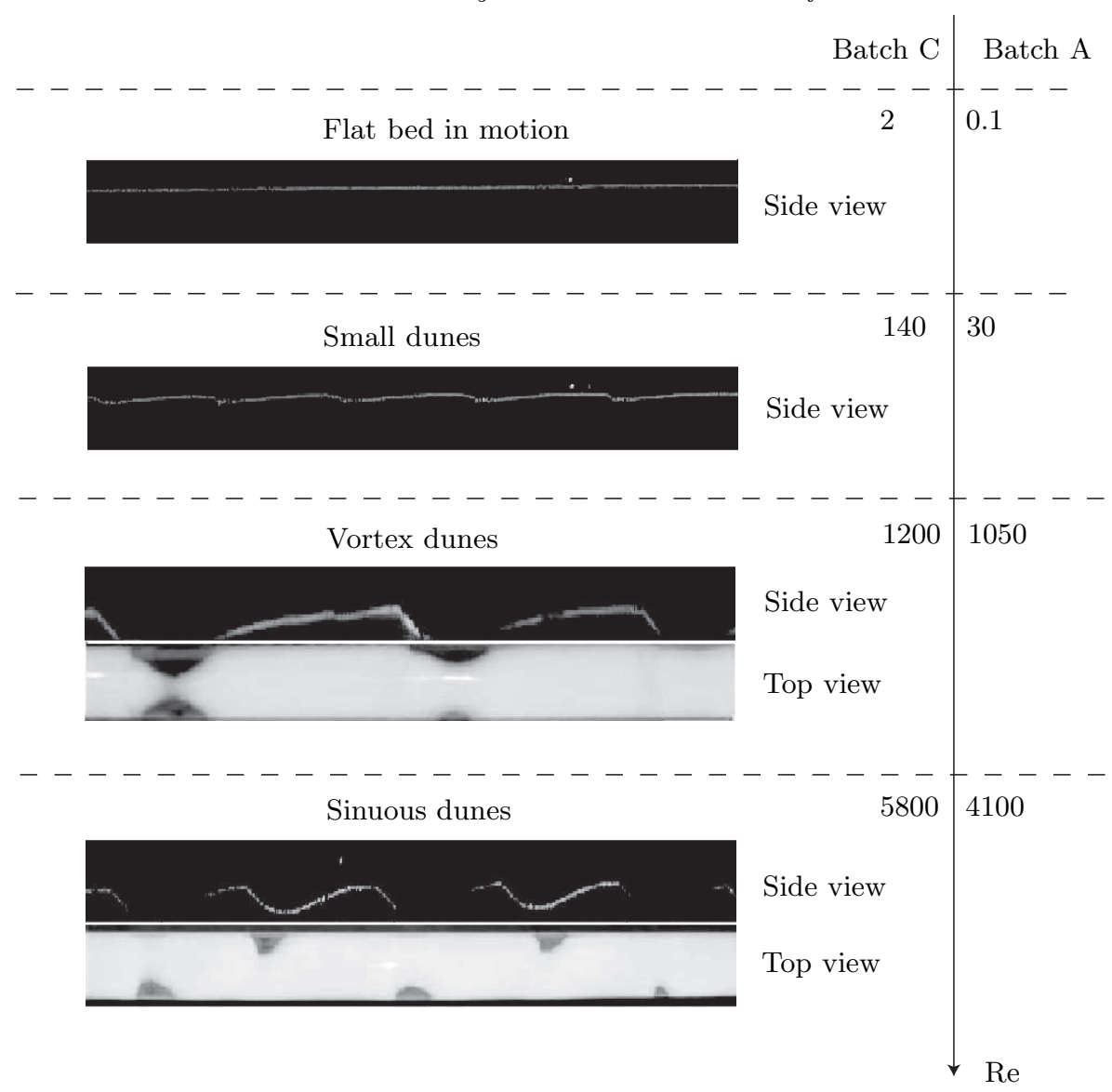

Figure 1. The different dune patterns.

increases with time whereas the dune velocity decreases. The dune motion eventually stops as the tube is not fed in with particles and the dunes are completely separated, see the bottom plots of figure 2 (b) and (c). We can note that the length-scale depends on the particles. The time-scale also differs. For batch $\mathrm{C}$ in fluid 1 , the dunes stop after less than one week, while, for batch $\mathrm{A}$ in fluid 1 with a flow rate of the same order of magnitude, they move during approximately two weeks before stopping.

In the case of the small dunes, there is also a propagation of dunes created by the entrance perturbation. But, as these dunes have no vortex, the velocities of dunes due to the entrance perturbation and of the initial dunes are similar and thus these dunes do not overtake the initial dunes created in the measurement zone, see the top plot of figure 2 (a). As time is increased, the wavelength increases without showing any saturation, the dune velocity decreases, and the dune amplitude increases, see the bottom plot of figure 2 (a). The small dunes never stop moving downstream and eventually leave the tube when they reach the tube outlet. When all the dunes have left the tube, the bed becomes flat again. A strong change in the pattern can be observed at the bottom of figure 2 (a) as a ripple instability is observed.

Small dunes and vortex dunes exhibit different behaviours. While the particle bed eventually freezes in both cases, their final states also differs. For vortex dunes, due to presence of vortices, the final state corresponds to immobile dunes separated by regions 


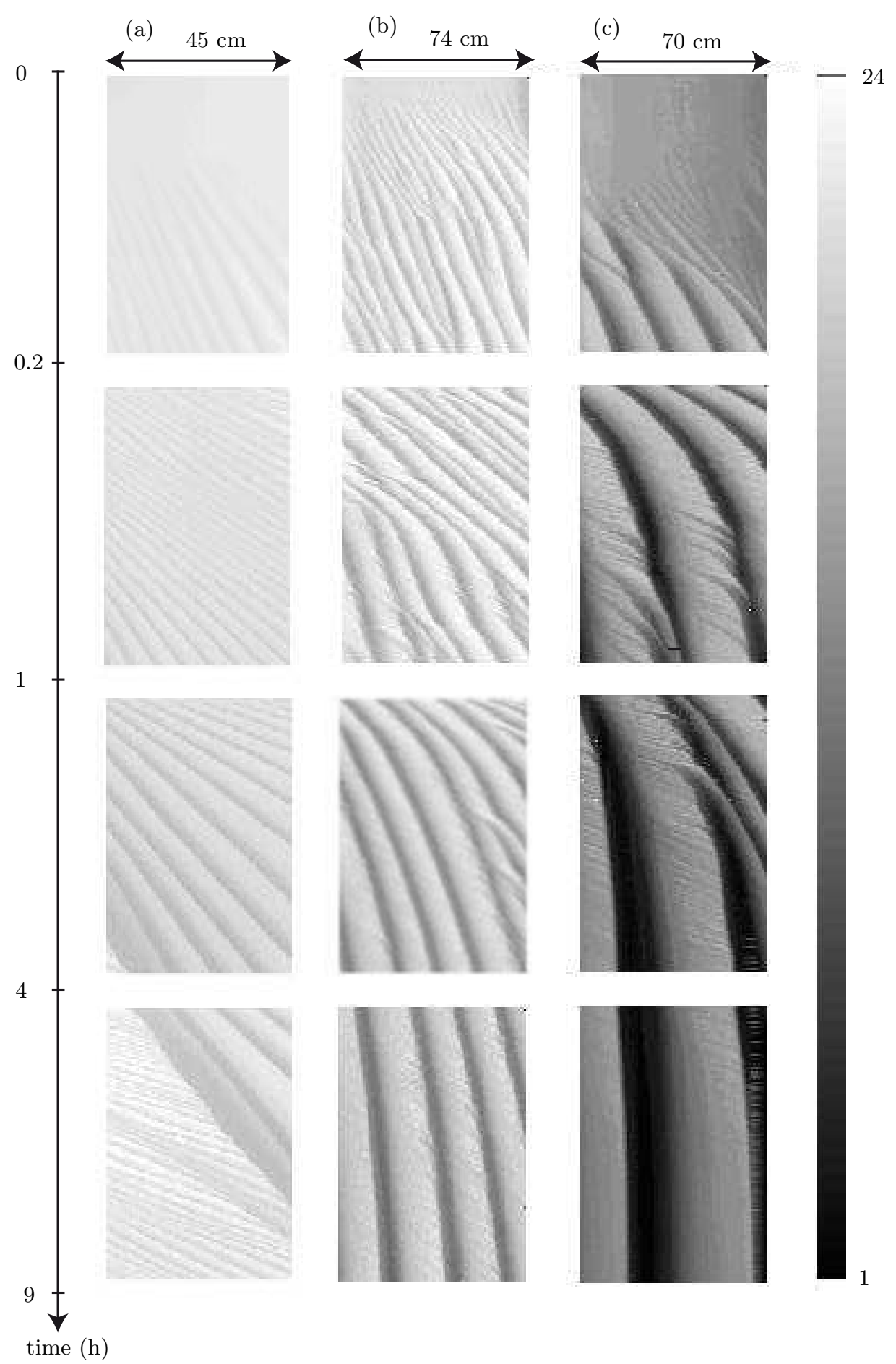

Figure 2. Spatio-temporal plots for (a) batch A in fluid 2 with $Q_{\text {pipe }}=3.4010^{-5} \mathrm{~m}^{3} \mathrm{~s}^{-1}$ and $h_{p}^{\text {start }}=22 \mathrm{~mm}$ (small dune regime), (b) batch A in fluid 1 with $Q_{\text {pipe }}=3.7910^{-5} \mathrm{~s}^{-1}$ and $h_{p}^{\text {start }}=21 \mathrm{~mm}$ (vortex dune regime), and (c) batch $\mathrm{C}$ in fluid 1 with $Q_{\text {pipe }}=3.9610^{-5} \mathrm{~m}^{3} \mathrm{~s}^{-1}$ and $h_{p}^{\text {start }}=15 \mathrm{~mm}$ (vortex dune regime). The grey scale represents the height $h_{p}$ of the bed in $\mathrm{mm}$. 

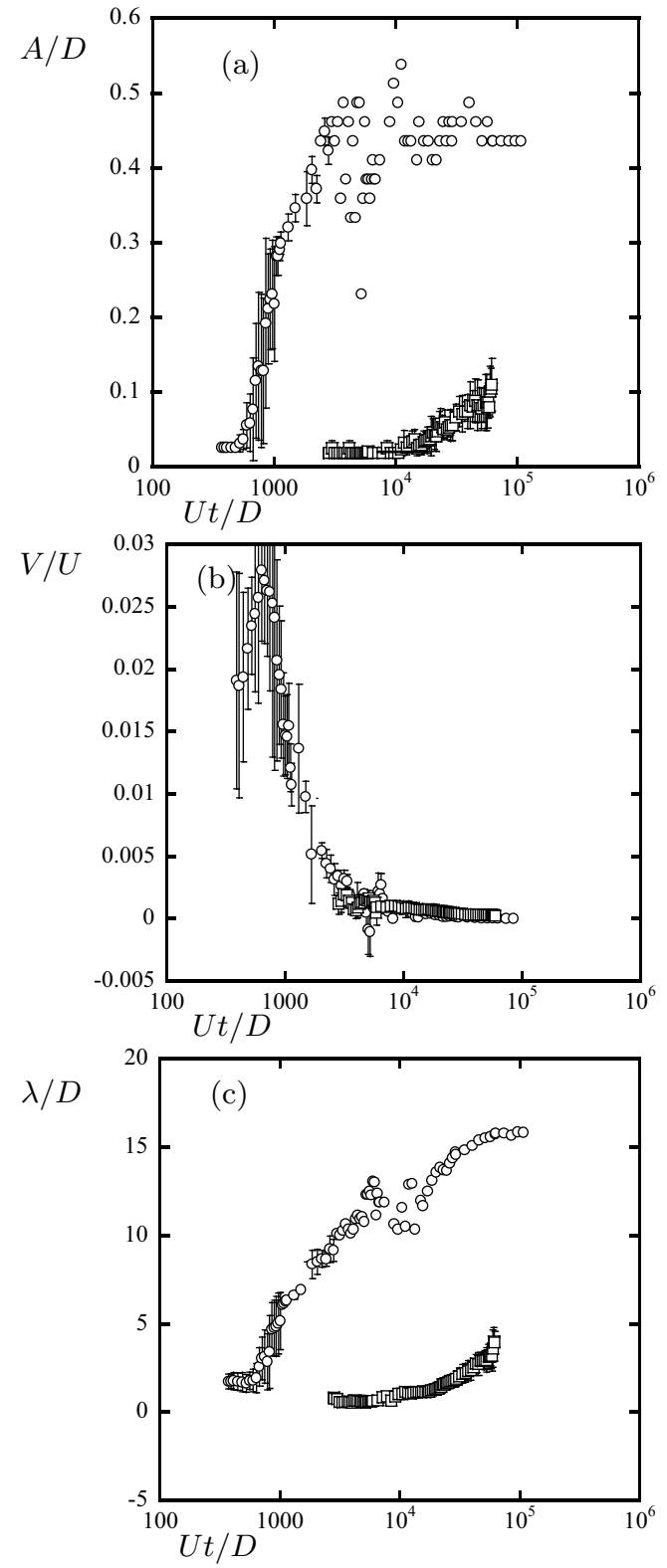

Figure 3. Dimensionless dune (a) amplitude, $A$, (b) velocity, $V$, and (c) wavelength, $\lambda$, for batch C in fluid 1 with $Q_{\text {pipe }}=3.9610^{-5} \mathrm{~m}^{3} \cdot \mathrm{s}^{-1}$ (o, vortex dune regime) and for batch A in fluid 4 with $Q_{\text {pipe }}=3.4610^{-5} \mathrm{~m}^{3} \cdot \mathrm{s}^{-1}(\square$, small dune regime)

emptied of particles. For small dunes, as there are no vortices to dig out the particle bed, the small dunes move downstream until they leave the pipe and the remaining state is a flat bed. These two behaviours are shown in online movies 2 and 3. Beside, their length-scales and time-scales are dissimilar even at the initial stage.

To obtain a quantitative comparison, we have measured the amplitude, wavelength, and phase velocity of the dunes in both regimes. The bed height measurements described above are further analysed to determine the local minima and maxima of the height 


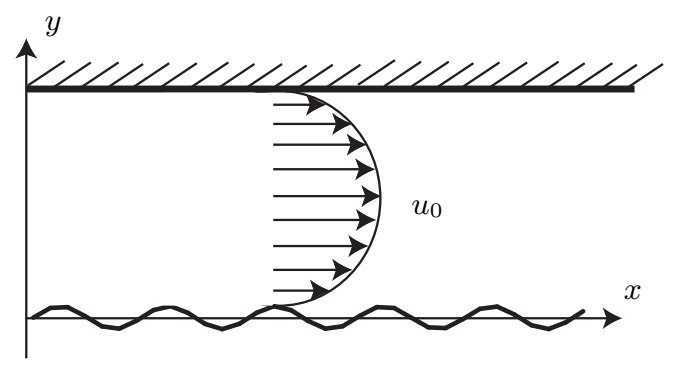

Figure 4. Poiseuille flow on a wavy bottom.

profile. For each acquisition time, the wavelength is given by the average of the distance between two maxima over the measurement zone. In a similar way, the amplitude is given by the averaged difference in height between the minima and maxima. The velocity is given by averaging the displacements of maxima over typically 5 acquisition times.

Figure 3 shows the evolution of amplitude, wavelength, and phase velocity with time of dunes appearing in the measurement zone in the small $(\square)$ and vortex (o) dune regimes. The quantities have been made dimensionless by using $D$ as a length scale and the mean velocity of the flow $U=4 Q_{\text {pipe }} / \pi D^{2}$ as a velocity scale. The time scale of dune formation differs by a factor ten between the two dune regimes. The amplitude and wavelength are similar in the first instant. However, the phase velocity of the initial dunes is about ten times larger in the vortex dune regime than in the small dune regime as shown in figure 3 (b). The behaviour also differs as it increases initially in the vortex dune regime while it presents a slow decrease in the small dune regime. This clearly shows that the dynamics of the initial dunes which precede the fully developed vortex dunes differ from those of the small dunes. At later instant, for a dimensionless time $\approx 900$ in figure 3 , there is a dramatic increase of both amplitude and wavelength as well as a maximum in the phase velocity in the vortex dune regime. This corresponds to the take-over of the fully developed vortex dune having vortices which intensify the dynamics. For further times, the amplitude and wavelength of the vortex dunes saturate as the dunes progressively become detached entities. Their speed decreases and the dunes eventually stop moving. Small dunes have an amplitude about five times smaller than vortex dunes at their later stage. Their wavelength shows a slow and continuous increase.

\section{A simple linear stability analysis}

\subsection{Poiseuille flow on a wavy bottom}

To determine the fluid flow over a wavy bottom in a two-dimensional channel, we follow the approach of Charru \& Hinch (2000) initially undertaken for a Couette flow that we adapt for a Poiseuille flow. We consider a fluid layer lying between a flat upper wall and a wavy bottom which is assumed to be perturbed sinusoidally as $\xi=\xi_{1} \cos (k x)$ with wave-number $k$ and amplitude $\xi_{1}$ as sketched in figure 4. Following the previous studies of Charru \& Hinch (2000) and Charru \& Hinch (2006), we assume that the time scale of the fluid flow is much shorter than the time scale of the bed evolution. The fluid flow can then be calculated as if the wavy bottom were fixed, by considering the superposition of a base flow, $u_{0}$ along the $x$ direction, over a flat bed and a disturbance, $u_{1}$ and $v_{1}$ along the $x$ and $y$ directions respectively, induced by the wavy bottom.

We decide to make all the values dimensionless by scaling the length by the channel thickness $h_{f}$, the velocity by $q_{f} / h_{f}$ where $q_{f}$ is the fluid flow rate, and the pressure by 
a viscous pressure $\eta_{f} q_{f} / h_{f}^{2}$. Therefore the time is scaled by $h_{f}^{2} / q_{f}$. We note $R e_{2 D}=$ $q_{f} / \nu$ the Reynolds number of the channel. The dimensionless velocity and pressure are decomposed into a base Poiseuille flow, $\bar{u}_{0}=6 \bar{y}(1-\bar{y})$ and $\partial \bar{p}_{0} / \partial \bar{y}=-\rho_{f} g h_{f}^{3} / \eta_{f} q_{f}$, and a disturbance $\left[\bar{u}_{1}(\bar{y}), \bar{v}_{1}(\bar{y}), \bar{p}_{1}(\bar{y})\right] e^{i \bar{k} \bar{x}}$. Here, the upper bar indicates dimensionless values, the 0 subscript the base state, and the 1 subscript the perturbation.

Substituting this flow into the dimensionless linearized mass and momentum conservation equations, we obtain a set of linear ordinary differential equations

$$
\begin{aligned}
i \bar{k} \bar{u}_{1}+\frac{\partial \bar{v}_{1}}{\partial \bar{y}} & =0 \\
\operatorname{Re}_{2 D}\left(i 6 \bar{k} \bar{u}_{1} \bar{y}(1-\bar{y})+6 \bar{v}_{1}(1-2 \bar{y})\right) & =-i \bar{k} \bar{p}_{1}-\bar{k}^{2} \bar{u}_{1}+\frac{\partial^{2} \bar{u}_{1}}{\partial \bar{y}^{2}} \\
i 6 \operatorname{Re}_{2 D} \bar{k} \bar{v}_{1} \bar{y}(1-\bar{y}) & =-\frac{\partial \bar{p}_{1}}{\partial \bar{y}}-\bar{k}^{2} \bar{v}_{1}-i \bar{k} \frac{\partial \bar{u}_{1}}{\partial \bar{y}^{2}} .
\end{aligned}
$$

The kinematic boundary conditions become

$$
\bar{u}_{1}(0)=-6 \bar{\xi}_{1} \quad \text { and } \quad \bar{u}_{1}(1)=\bar{v}_{1}(1)=\bar{v}_{1}(0)=0 .
$$

Equations (3.1) with the boundary conditions (3.2) can be solved numerically using a Chebychev spectral collocation method, see Gottlieb, Hussaini \& Orszag (1984). It is also interesting to find an analytical solution in the shallow viscous regime, $\bar{k} \ll$ 1 , by performing an asymptotic expansion for the small dimensionless wave-number $\bar{k}$. Assuming $R e_{2 D}=O(1)$, the fluid velocity components can be expended in powers of $\bar{k}$ which gives the shear-rate

$$
\frac{d \bar{u}_{1}}{d \bar{y}}=-6 \bar{\xi}_{1}(6 \bar{y}-4)+i \bar{\xi}_{1} \frac{R e_{2 D}}{70}\left(504 \bar{y}^{5}-1260 \bar{y}^{4}+840 \bar{y}^{3}-108 \bar{y}+12\right) \bar{k}+O\left(\bar{k}^{2}\right) .
$$

This exhibits the phase-lag of the bottom shear rate disturbance $\bar{k} R e_{2 D} / 140$ due to inertia similar to that found by Charru \& Hinch (2000) for Couette flow.

\subsection{Dune formation}

The dune growth is determined by the conservation equation for the particle flux, $q_{p}$, which is obtained by integrating the particle mass conservation equation over the bed height

$$
\frac{\partial \bar{q}_{p}}{\partial \bar{x}}+\phi_{0} \frac{\partial \bar{\xi}}{\partial \bar{t}}=0
$$

where $\phi_{0}$ is the particle volume fraction inside the bed.

We assume that the dynamics of the particle is well accounted by the algebraic law relating the dimensionless particle flux to the Shields number found by Ouriemi, Aussillous \& Guazzelli (2009)

$$
\bar{q}_{p}=\phi_{0} \frac{\eta}{\eta_{e}} \frac{G a}{24 R e_{2 D}} \frac{\theta^{3}}{\theta^{c^{2}}},
$$

where $G a=\rho_{f} \Delta \rho g d^{3} / \eta^{2}$ is the Galileo number, $\theta=(\eta d u / d y) / \Delta \rho g d$ the Shields number, and $\eta_{e}$ an effective viscosity of the mixture of the particles and fluid that was found to equate well to the Einstein viscosity $\eta\left(1+5 / 2 \phi_{0}\right)$. This algebraic law has been shown to be valid for moderate Shields numbers $0.5 \lesssim \theta \lesssim 1.5$, i.e above the threshold for incipient motion (the moving thickness is larger than one particle diameter) but for shearing flow not substantially perturbed by the motion of the granular media, see Ouriemi, Aussillous \& Guazzelli (2009). 
We now introduce the time evolution of the bed surface, $\bar{\xi}=\bar{\xi}_{1} e^{i(\bar{k} \bar{x}-\bar{\omega} \bar{t})}$. We decompose the Shields number into a base Shields number $\theta_{0}$ corresponding to a flat bed and a perturbation $\theta_{1} e^{i(\bar{k} \bar{x}-\bar{\omega} \bar{t})}$. In the same way, we write the critical Shields number as $\theta^{c}=$ $\theta_{0}^{c}+\theta_{1}^{c} e^{i(\bar{k} \bar{x}-\bar{\omega} \bar{t})}$. Linearising equation (3.5), we obtain

$$
\bar{q}_{p}=\frac{\eta}{\eta_{e}} \phi_{0} \frac{G a}{24 R e_{2 D}} \frac{\theta_{0}^{3}}{\theta_{0}^{c^{2}}}\left(1+3 \frac{\theta_{1} e^{i(\bar{k} \bar{x}-\bar{\omega} \bar{t})}}{\theta_{0}}-2 \frac{\theta_{1}^{c} e^{i(\bar{k} \bar{x}-\bar{\omega} \bar{t})}}{\theta_{0}^{c}}\right) .
$$

The local inclination of the bed surface modifies the critical Shields number, see e.g Fredsøe (1974), Richards (1980), Charru \& Hinch (2006), and Charru (2006), which becomes

giving by identification

$$
\theta^{c}=\theta_{0}^{c}\left(1+\frac{\partial \bar{\xi} / \partial x}{\mu}\right)=\theta_{0}^{c}+\frac{\theta_{0}^{c}}{\mu} i \bar{k} \bar{\xi}
$$

$$
\theta_{1}^{c}=\frac{\theta_{0}^{c}}{\mu} i \bar{k} \bar{\xi}_{1}
$$

where $\mu$ is a friction coefficient which is the tangent of the angle of repose of the grains and mainly depends on the grain geometry.

We suppose that the time evolution of the bed surface is slow enough to relate the Shields number to the shear rate calculated at the top of the fixed wavy bottom found in the preceding section

$$
\theta=\frac{R e_{2 D}}{G a}\left(\frac{d}{h_{f}}\right)^{2}\left[\frac{d \bar{u}_{0}}{d \bar{y}}(\bar{\xi})+\frac{d \bar{u}_{1}}{d \bar{y}}(\bar{\xi}) e^{i(\bar{k} \bar{x}-\bar{\omega} \bar{t})}\right]
$$

The linearized shear rate calculated at the top of the fixed wavy bottom is given by the two equations

$$
\begin{array}{r}
\frac{d \bar{u}_{0}}{d \bar{y}}(\bar{\xi})=\frac{d \bar{u}_{0}}{d \bar{y}}(0)+\bar{\xi} \frac{d^{2} \bar{u}_{0}}{d^{2} \bar{y}}(0)+O\left(\bar{\xi}^{2}\right), \\
\frac{d \bar{u}_{1}}{d \bar{y}}(\bar{\xi})=\frac{d \bar{u}_{1}}{d \bar{y}}(0)+O\left(\bar{\xi}^{2}\right),
\end{array}
$$

exhibiting

$$
\begin{array}{r}
\theta_{0}=\frac{R e_{2 D}}{G a}\left(\frac{d}{h_{f}}\right)^{2} \frac{d \bar{u}_{0}}{d \bar{y}}(0), \\
\theta_{1}=\frac{R e_{2 D}}{G a}\left(\frac{d}{h_{f}}\right)^{2}\left[\bar{\xi}_{1} \frac{d^{2} \bar{u}_{0}}{d^{2} \bar{y}}(0)+\frac{d \bar{u}_{1}}{d \bar{y}}(0)+O\left(\bar{\xi}_{1}^{2}\right)\right] .
\end{array}
$$

Considering the time evolution of the bed surface and introducing the linearized flowrate given by equation (3.6), the conservation equation of the particle flux at leading order in $\bar{\xi}_{1}$ becomes

$$
\bar{\omega} \bar{\xi}_{1}=\bar{k} \frac{\eta}{\eta_{e}} \frac{G a}{24 R e_{2 D}} \frac{\theta_{0}^{3}}{\theta_{0}^{c^{2}}}\left(3 \frac{\theta_{1}}{\theta_{0}}-2 \frac{\theta_{1}^{c}}{\theta_{0}^{c}}\right) .
$$

Clearly, the perturbation in critical Shields number due to the local inclination of the bed surface (which is purely imaginary) has always a stabilising effect while the imaginary part of the perturbation in Shields number contains the destabilising effect due to inertia. The frequency $\bar{\omega}$ is directly given by equation (3.12) where $\theta_{1}$ is obtained using the numerical computation of equations (3.1) with the boundary conditions (3.2) mentioned in the preceding section. The frequency can be separated into an imaginary part and a real part, giving the growth rate $\bar{\omega}_{i}$ and the phase velocity $\bar{\omega}_{r} / \bar{k}$. 

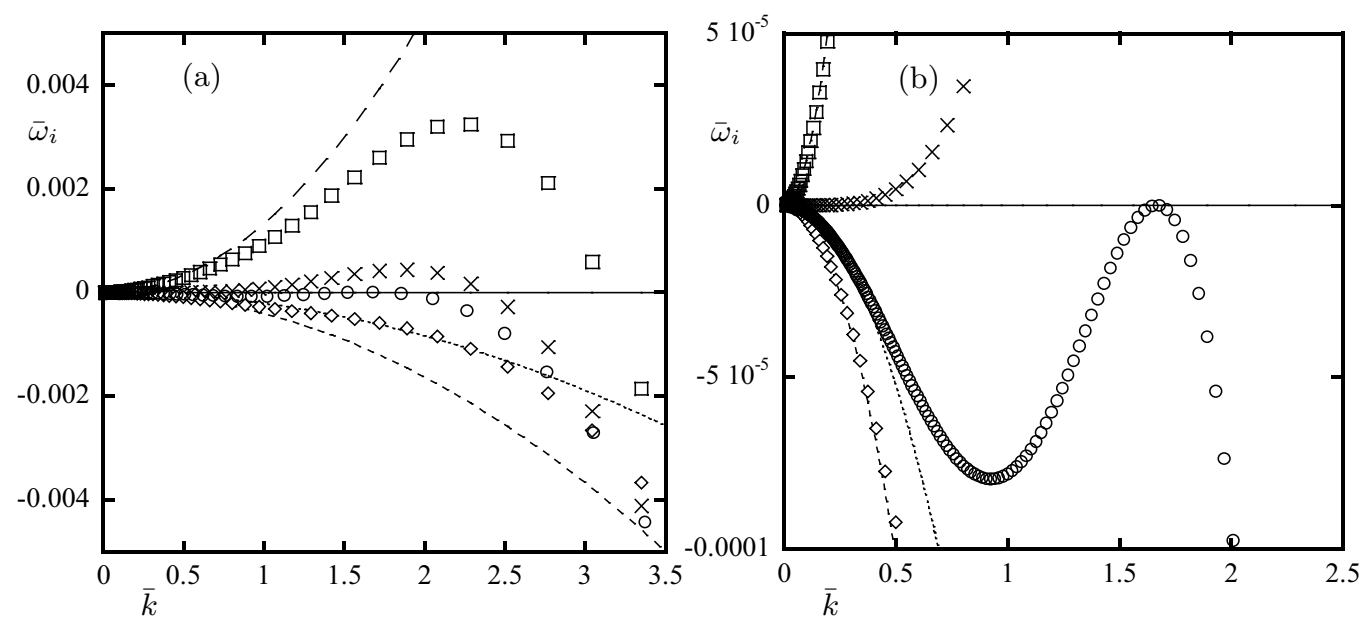

Figure 5. (a) Numerical $\bar{\omega}_{i}$ as a function of $\bar{k}$ for $R e_{2 D}=35(\diamond), R e_{2 D}=49.3(\circ)$, $R e_{2 D}=54.3(\times)$, and $R e_{2 D}=70(\square)$. The lines represent equation (3.15) for $R e_{2 D}=35$ (small-dashed line), $R e_{2 D}=49.3$ (dotted line), $R e_{2 D}=54.3$ (solid line), and $R e_{2 D}=70$ (long-dashed line) and (b) blow-up.

In the shallow viscous regime, $\bar{k} \ll 1$, equation (3.12) can be solved analytically by using the shear rate given by equation (3.3). At leading order in $\bar{k}$, this gives

$$
\bar{\omega}=\bar{k} \frac{\eta}{\eta_{e}} \frac{G a}{24 \operatorname{Re}_{2 D}} \frac{\theta_{0}^{3}}{\theta_{0}^{c^{2}}}\left(6+i \frac{3 R e_{2 D}}{35} \bar{k}-2 i \bar{k} \frac{1}{\mu}\right),
$$

which leads to

$$
\begin{array}{r}
\frac{\bar{\omega}_{r}}{\bar{k}}=\frac{\eta}{\eta_{e}} \frac{G a}{4 R e_{2 D}} \frac{\theta_{0}^{3}}{\theta_{0}^{c^{2}}}, \\
\bar{\omega}_{i}=\bar{k}^{2} \frac{\eta}{\eta_{e}} \frac{G a}{24 R e_{2 D}} \frac{\theta_{0}^{3}}{\theta_{0}^{c^{2}}}\left(\frac{3 R e_{2 D}}{35}-2 \frac{1}{\mu}\right) .
\end{array}
$$

The instability threshold, corresponding to $\omega_{i}=0$, occurs at

$$
\operatorname{Re}_{2 D}^{c}=\frac{70}{3 \mu}
$$

The important finding is that the control parameter for the dune instability is the Reynolds number, $R_{2} D$, and not the Shields number, $\theta$. It is worth mentioning that the threshold for having a monolayer of particles in motion on a flat bed has been found to be $\theta_{0}^{c}=\mu \phi_{0} / 2$ which is proportional to the friction coefficient $\mu$ and to the volume fraction of the particle inside the bed $\phi_{0}$, see Ouriemi, Aussillous \& Guazzelli (2009). The threshold for dune instability involves the Reynolds number and is also related to the friction coefficient. Using the above expression for $\theta_{0}^{c}$, it can be expressed as

$$
R e_{2 D}^{c}=\frac{35 \phi_{0}}{3 \theta_{0}^{c}}
$$

These thresholds, $\theta_{0}^{c}$ and $R e_{2 D}^{c}$, differ but are related. Consequently, the onset for the instability may not coincide with the onset for particle motion. In this simple modelling, these thresholds only depend on two physical parameters, the particle volume fraction inside the bed and the friction coefficient.

Figure 5 shows the dimensionless growth-rate, $\bar{\omega}_{i}$, versus dimensionless wave-number, 


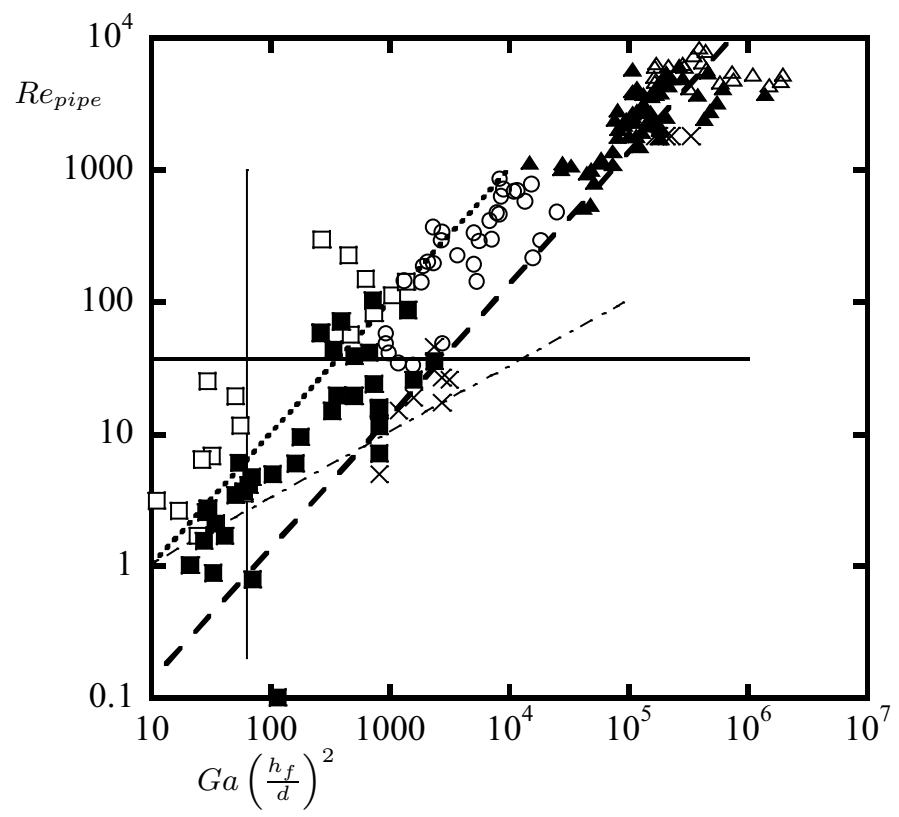

Figure 6. Phase-Diagram of the dune patterns in the plane $R e_{\text {pipe }}, G a\left(h_{f} / d\right)^{2}$. The different regimes represented are: 'no motion' $(x)$, 'flat bed in motion' $(\boldsymbol{\square})$ and $(\square)$ when outside the domain of validity of the model, 'small dunes' (o), 'vortex dunes' ( $\mathbf{\Lambda})$, and 'sinuous dunes' $(\triangle)$. The dashed line is the predicted threshold for particle motion, the horizontal solid line is the predicted instability threshold, and the dotted line indicates the domain of validity of the particle flux law. The dashed-dotted line is the prediction for instability threshold of Charru \& Mouilleron-Arnould (2002) and the vertical solid line is that of Charru \& Hinch (2006).

$\bar{k}$, for $\phi_{0}=0.55$ and $\theta_{0}^{c}=0.12$ as found experimentally by Ouriemi et al. (2007) (or equivalently $\mu=0.43)$. Equation (3.15) valid in the shallow viscous regime, $\bar{k} \ll 1$, shows a long wavelength instability with a threshold at $\operatorname{Re}_{2 D}^{c}=54.3$. The numerical predictions present a good agreement with this asymptotic case for $\bar{k} \lesssim 0.1$. However, the numerical solution indicates that the instability is not a long-wave instability at threshold but presents a finite value $\bar{k} \approx 1.7$ for a slightly different threshold $R e_{2 D}^{c}=49.3$, see blow-up in figure 5. This is an interesting finding as it indicates that the wavelength at onset is of the order of the fluid thickness.

\section{Discussion and conclusion}

Figure 6 presents the phase diagram of the dune patterns in the plane $R e_{\text {pipe }}, G a\left(h_{f} / d\right)^{2}$. We choose this plane to exhibit both the threshold for incipient particle motion controlled by the Shields number and that for dune instability predicted to be controlled by the Reynolds number in the linear stability analysis of $\S 3$. In this plane, the threshold for particle motion is given by the dashed line $R e_{\text {pipe }}=\left(2 \theta_{0}^{c} / 3 \beta \pi\right) G a\left(h_{f} / d\right)^{2} \approx 0.014 G a\left(h_{f} / d\right)^{2}$ with $\theta_{0}^{c}=0.12$ as found experimentally by Ouriemi et al. (2007) with the same experimental apparatus. The predicted instability threshold is the horizontal solid line $R e_{\text {pipe }}=140 \phi_{0} / 3 \beta \pi \theta_{0}^{c} \approx 37$. Indeed, in the case of a pipe flow, the Reynolds number is $R e_{\text {pipe }}=4 \rho_{f} Q_{\text {pipe }} / \pi \eta D$ and the threshold equation (3.17) obtained for a two dimensional channel needs to be modified by replacing $\operatorname{Re}_{2 D}$ by $\beta \pi R e_{\text {pipe }} / 4$ (or equivalently $q_{f}$ by $\left.\beta Q_{\text {pipe }} / D\right)$ where $\beta=1.85$ has been found from numerical analysis in the limit 

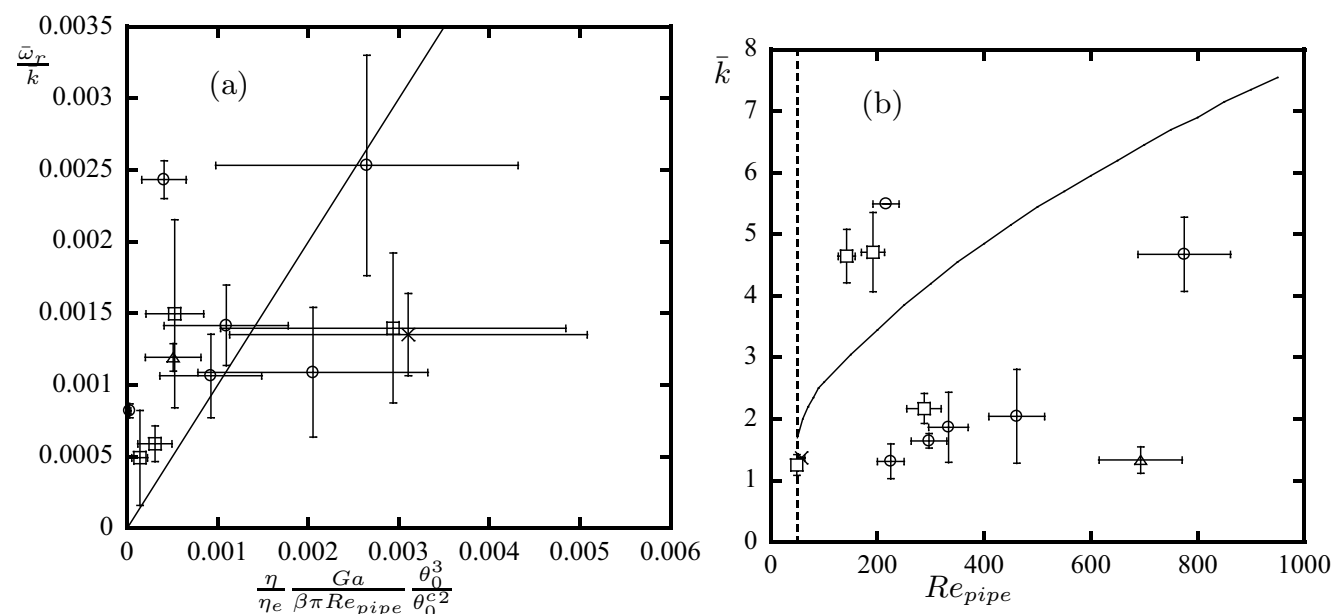

Figure 7. (a) Dimensionless initial dune velocity against equation (3.14) (the solid line corresponds to the slope one) and (b) dimensionless initial wavenumber as a function of $R e_{\text {pipe }}$ (the solid line represents the numerical prediction of the most unstable wave-number and the dotted line the instability threshold) for batch $\mathrm{A}$ in fluids 2 (o), $3(\triangle), 4$ $(\square)$, and $5(\times)$.

$0.2 \leqslant h_{f} / D \leqslant 0.8$, see Ouriemi et al. (2007). Note that this predicted threshold only depends on a single parameter $\left(\mu\right.$ or $\left.\theta_{0}^{c}\right)$. The dotted line indicates the domain of validity of the algebraic law relating the dimensionless particle flux to the Shields number found by Ouriemi, Aussillous \& Guazzelli (2009) and thus indicates the domain of validity of the instability threshold prediction of $\S 3$. The three regimes of 'no motion' $(\times)$, 'flat bed in motion' ( $\square$ and $\square$ when outside the domain of validity of the model), and 'small dunes' (o) are well delineated by these boundaries in the given limit of validity. Clearly, the threshold for incipient particle motion and that for small dune instability are observed to differ as there is a large region of 'flat bed in motion' without any dune formation. The instability threshold is well described by $R e_{\text {pipe }}$ as a control parameter and not by $\theta$ which would be a line parallel to the dashed line nor by $G a$ which would be a vertical line in figure 6 . The threshold prediction of the simple linear stability analysis of $\S 3$ (horizontal solid line $R e_{\text {pipe }} \approx 37$ ) provides a correct boundary for the 'small dune' instability. Furthermore, the regimes of 'vortex dunes' $(\boldsymbol{\Lambda})$ and 'sinuous dunes' $(\triangle)$ seem separated and their thresholds also well described by $R e_{\text {pipe }}$ as a control parameter.

We have also tested in figure 6 the prediction for instability threshold of Charru \& Mouilleron-Arnould (2002) (dashed-dotted line) and Charru \& Hinch (2006) (vertical solid line) listed in table 1 . Below both of these predicted thresholds, no dune is observed as expected but these boundaries do not properly delimitate the regime of small dune formation. Note that we did not calibrate the adjustable coefficients of these two models but used the original calibration of Charru \& Mouilleron-Arnould (2002) and Charru \& Hinch (2006) respectively. Different coefficients would produce boundaries having the same slope as the dashed-dotted and vertical solid lines. These would still be unable to describe the small dune threshold in figure 6 . The difference between the stability analysis of $\S 3$ and that of Charru \& Mouilleron-Arnould (2002) lies into the algebraic law relating $q_{p}$ to $\theta$. The analysis of $\S 3$ uses $q_{p} \propto \theta^{3} / \theta^{c 2}$ while Charru \& Mouilleron-Arnould (2002) takes $q_{p} \propto\left(\theta-\theta^{c}\right)^{3}$. Using a power law involving the excess Shields number yields a threshold depending both on the Reynolds and Galileo numbers (see table 1) and having the slope of the dashed-dotted line in figure 6. The analysis of Charru \& Hinch (2006) 
differs from the present analysis by the use of a different model for particle transport which is meant to be applied only to a mobile monolayer and which introduces the new stabilising effect of crest erosion. The competition between this new stabilising effect and the destabilising fluid inertia depends on the Galileo number. If the Galileo number is smaller than a critical number (given in table 1 and vertical solid line plotted in figure 6), crest erosion overcomes and the bed is stable. In the opposite case, the bed is unstable above a critical Shields number. This last threshold would correspond to a line with a slope similar to that of the dashed line in figure 6 which clearly does not delimitate the observed instability.

Further quantitative comparisons with the predictions of the stability analysis of $\S 3$ can be attempted for the onset of small dunes. Figure 7 (a) compares the initial dune velocity with equation (3.14). Despite large error bars and some dispersion of the data, the agreement is good. Figure 7 (b) presents the initially observed wave-numbers as well as the most amplified numerical wavenumber as a function of $R e_{\text {pipe }}$. Clearly, a long-wave instability is not observed in the experiments and a finite value $\approx 1.2 h_{f}^{-1}$ is obtained at threshold. The experimental wave-numbers seem rather independent of $R e_{\text {pipe }}$ with values $\approx 1-5 h_{f}^{-1}$ while the numerical wavenumber is $\approx 1.7 h_{f}^{-1}$ at threshold and presents an increase with increasing $R e_{\text {pipe }}$. Nonetheless, same order of magnitude is recovered close to threshold. It should be, however, mentioned that experimental measurements at instability onset are very delicate and thus do not permit further detailed comparisons. Note that, as expected, the stability analysis developed here is unable to account for the onset of the vortex dunes. Equation (3.14) underestimates by a factor $10^{4}$ the experimental velocity of the vortex dunes. Note also that, while in figure 6 , the experimental data for small dunes are obtained with particles of batches $\mathrm{A}$ to $\mathrm{C}$ with fluids 1 to 5 , in figure 7 only the data for particles of batch A and fluids 2 to 5 are used.

In conclusion, we have given the experimental phase diagram of the different dune patterns observed when a bed composed of spherical particles is submitted to a shearing flow in a pipe. 'Small dunes' present small amplitudes and only exist in laminar flow. 'Vortex dunes' are characterised by the existence of vortices at their front and are found either in laminar or turbulent flow. 'Sinuous dunes', showing a double periodicity, appear in turbulent flow. While the threshold for incipient motion is determined by the Shields number, that for dune formation seems to be described by the Reynolds number and not by the Shields or Galileo numbers. Moreover, the dune instability is not a longwave instability at threshold but do present a finite vawelength of the order of the fluid thickness.

To predict the small dune formation, we have performed a simple linear stability analysis where inertia in the fluid produces a phase-lag in the shear stress which is destabilising, while the component of gravity down an incline stabilises the perturbations. We first calculated the perturbed fluid flow over a wavy bottom considered as if fixed. Then, we used the particle flux found by Ouriemi, Aussillous \& Guazzelli (2009) to relate the bed height evolution to the shear stress at the top of the bed through the particle mass conservation. The threshold for dune formation is found to be controlled by the Reynolds number. This threshold prediction with a single adjustable parameter that we have taken to be realistic provides a correct boundary for the onset of 'small dunes'. The predicted wavelength at instability threshold is of the order of the fluid thickness in agreement with the experiments.

This simple stability analysis containing the basic ingredient of the destabilising fluid inertia and stabilising gravity is found sufficient to provide realistic predictions. It is worth pointing out that the two-phase nature of the problem has been only accounted for in the particle conservation equation in the present study and in most of the studied found in the 
literature. The interesting questions are whether there is any delay in the flux adapting to a change in the shear stress and whether this additional stabilising mechanism as well as particle inertia and feed back production are significant. Undertaking a stability analysis using the complete two-phase modelling developed by Ouriemi, Aussillous \& Guazzelli (2009) should enable to answer these issues in the future.

\section{Acknowledgement}

We would like to thank Y. Forterre and O. Pouliquen for discussions regarding the dune stability analysis, F. Charru and E. J. Hinch for comments and critical reading, and P. Cervetti, S. Martinez, and F. Ratouchniak for technical assistance. Funding from the Institut Français du Pétrole and Agence Nationale de la Recherche (Project Dunes ANR07-3_18-3892) are gratefully acknowledged. This work is part of the thesis of M. Ouriemi on 'Érosion, transport et instabilités d'un lit de particules dans un tube' (Université de Provence, 2007).

\section{REFERENCES}

Andreotti, B., Claudin, P. \& Douady, S. 2002 Selection of dune shapes and velocities. Part 2: a two-dimensional modelling. Eur. Phys. J. B 28, 341-352.

Charru, F. \& Hinch, E. J. 2000 'Phase diagram' of interfacial instabilities in a two-layer Couette flow and mechanism of the long-wave instability. J. Fluid Mech. 414, 195-223.

Charru, F. \& Hinch, E. J. 2006 Ripple formation on a particle bed sheared by a viscous liquid. J. Fluid Mech. 550, 111-121.

Charru, F. \& Mouilleron-Arnould, H. 2002 Instability of a bed of particles sheared by a viscous flow. J. Fluid Mech. 452, 303-323.

Charru, F., Moullleron-Arnould, H. \& Eiff, O. 2004 Erosion and deposition of particles on a bed sheared by a viscous flow. J. Fluid Mech. 519, 55-80.

Charru, F. 2006 Selection of the ripple length on a granular bed sheared by a liquid flow. Phys. Fluids. 18, 121508.

Claudin, F. \& Andreotti, B. 2006 A scaling law for aeolian dunes on Mars, Venus, Earth, and for subaqueous ripples. Earth and Planetary Science Letters. 252, 30-44.

Colombini, M. 2004 Revisiting the linear theory of sand dune formation. J. Fluid Mech. 502, $1-16$.

Engelud, F. 1970 Instability of erodible beds. J. Fluid Mech. 42, 225-224.

FREDSøE, J. 1974 On the development of dunes in erodible channels. J. Fluid Mech. 64, 1-16.

Gottlieb, D., Hussaini, M. Y.\& Orszag, S. A. 1984 Theory and application of spectral methods. In Spectral Methods for Partial Differential Equations (ed. R. G. Voight, D. Gottlieb \& M.Y. Hussaini). SIAM.

KenNedy, J. F. 1963 The mechanics of dunes and antidunes in erodible-bed channels. J. Fluid Mech 16, 521-544.

Loiseleux, T., Gondret, P., Rabaud, M., \& Doppler, D. 2005 Onset of erosion and avalanches for an inclined granular bed sheared by a continuous laminar flow. Phys. Fluids $\mathbf{1 7}, 103304$.

Ouriemi, M., Aussillous, P., Medale, M., Peysson, Y. \& Guazzelli, E. 2007 Determination of the critical Shields number for particle erosion in laminar flow. Phys. Fluids 19, 061706.

Ouriemi, M., Aussillous, \& Guazzelli, E. 2009 Sediment dynamics. Part 1. Bed-load transport by shearing flows. J. Fluid Mech.

RichARDS, K. 1980 The formation of ripples and dunes on an erodible bed. J. Fluid Mech. 99, 597-618.

Sumer, M. \& BAKioglu, M. 1984 On the formation of ripples on an erodible bed. J. Fluid Mech. 144, 177-190. 